\title{
A survey on the risk factors for the prevalence of dental caries among preschool children in Japan
}

\author{
Yoshiaki Nomura ${ }^{1,2)}$, Shinpei Tsuge ${ }^{3)}$, Masaki Hayashi ${ }^{3)}$, Masahiro Sasaki ${ }^{3)}$, \\ Tetsuya Yamauchi ${ }^{3)}$, Nobuyoshi Ueda ${ }^{3)}$ and Nobuhiro Hanada ${ }^{1)}$ \\ 1) Department of Oral Health, National Institute of Public Health \\ 1-23-1 Toyama, Shinjuku-ku, Tokyo 162-8640, JAPAN \\ ${ }^{2)}$ Department of Preventive Dentistry and Public Health, Tsurumi University School of Dental Medicine \\ 2-1-3 Tsurumi, Tsurumi-ku, Yokohama 230-8501, JAPAN \\ 3) Gifu Dental Association \\ 1-8 Kano-Jounan-dori, Gifu-shi, Gifu 500-8486, JAPAN
}

\begin{abstract}
The etiology of dental caries has been suggested to be multifactorial. We conducted a cross-sectional study to investigate the contribution of the risk factors for dental caries, surveying a total of 645 preschool children at medical check-ups. Among 10 factors investigated in this study, salivary flow, amount of Lactobacillus, amount of total Streptococci, amount of mutans streptococci, and daily number of times of sweet soft drinks correlate with the prevalence of dental caries. Multivariate Logistic regression analysis using the five factors that correlated produced only two factors, salivary levels of mutans streptococci and Lactobacillus, which correlated with the prevalence of dental caries. Furthermore, clear dose-response relationships were observed in these two factors. We therefore suggest that cariogenic bacteria are the most important risk factor for dental caries among preschool children in Japan.
\end{abstract}

\author{
Key words \\ Lactobacilli, \\ Mutans streptococci, \\ Preschool children, \\ Risk factors, \\ Saliva
}

\section{Introduction}

Dental caries has been suggested to be a multifactorial disease. Keyes has proposed three main factors in the etiology of dental caries: host, substrate, and microflora ${ }^{1)}$. Furthermore, the mechanism of dental caries was theoretically explained ${ }^{2}$. Some clinical trials have shown that controlling these factors suppresses the incidence of dental caries. For the host factors, sodium fluoride has been suggested as useful for preventing dental caries both clinically and economically ${ }^{3}$. For the substitute factors, diet sugar consumption is correlated with the incidence of new dental caries ${ }^{4,5}$, and restricting the intake of diet sugar has been suggested to reduce the incidence of dental caries ${ }^{6}$. Recently, xylitol has been shown to be useful as an alternative sucrose, and its efficiency was confirmed ${ }^{7}$. For microflora

Received on September 22, 2003

Accepted on January 14, 2004 factors, plaque control has conventionally been used as the primary preventive method. Many clinicians have used plaque control as a tool for fighting both dental caries and also periodontal disease. Furthermore, application of anti-microbial drugs could reduce the number of mutans streptococci (Streptococcus mutans and Streptococcus sobrinus) in saliva or plaque for a few months ${ }^{8}$.

These factors are all correlated with the etiology of dental caries. However, the weight of these factors contributing to the prevalence or incidence of dental caries has not been clarified. These factors may confound each other in attempts to explain the etiology of dental caries, and some studies have shown that controlling only one of these factors could not suppress the incidence of dental caries completely.

In this study, we obtained clinical samples and information about the etiology of dental caries by questionnaires at preschool medical check-ups. The aim of this study was to analyze the contribution 

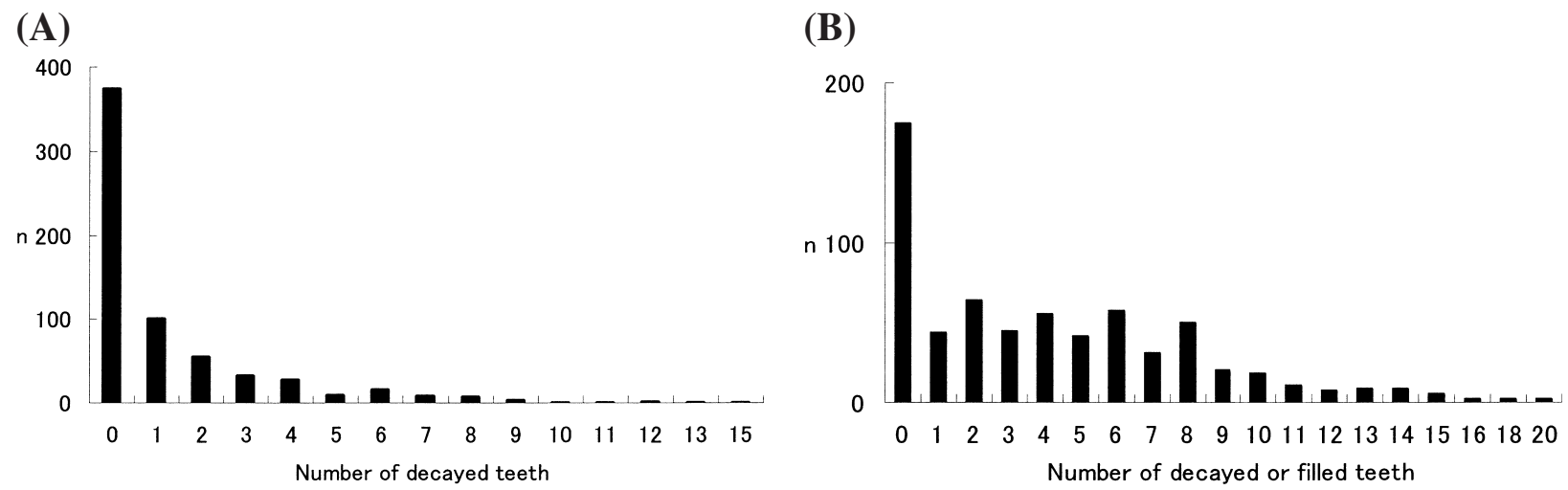

Fig. 1 The distribution of $\mathrm{dt}$ and $\mathrm{dft}$

The distribution of the number of decayed deciduous teeth (A) and decayed or filled teeth (B) in this study. The median of the decayed teeth was 0 and decayed or filled teeth was 3 . With the erupted permanent teeth, no decayed of filled teeth was observed.

of these factors in children by multiple Logistic regression analysis to clarify their importance.

\section{Materials and methods}

\section{Study population}

The study population was sampled from preelementary school children (five or six years old) residing in the Ena and Nakatsugawa areas of Gifu Prefecture, Japan. The fluoride concentration of drinking water in this area is less than $0.8 \mathrm{ppm}$. Thirteen of the thirty elementary schools, 9 from Ena area and 4 from Nakatsugawa, were selected to sample the population. Children were informed of the survey by the letter from the municipal office announcing their entry into elementary school and a total of 645 children participated in this study. Twelve children dropped out, primarily because of relocation and missing check ups due to illness. We obtained clinical samples and questionnaires during the preschool medical check-ups; we obtained informed consent at the collection of the questionnaires.

\section{Clinical examination and clinical samples}

Dentists conducted oral examinations under a light and using dental mirrors. Teeth examined for dental caries were scored as sound, decayed or filled. The decayed or filled teeth were identified according to the WHO standard method and criteria ${ }^{9}$.

Saliva samples were obtained by having subjects chew a gum base that contains no taste or flavor additives for 3 mins. The 3-min stimulated salivary flow and salival buffering capacity were evaluated by $\mathrm{pH}$ testing paper (Toyoroshi, Tokyo, Japan).

\section{Microbial procedures}

To quantify the total Streptococci, mutans streptococci, and Lactobacilli in saliva, we performed microbial procedures according to the method described previously ${ }^{10)}$. Saliva samples $(50 \mu l)$ were sonicated by ultrasonic dispersion (60 power output) for 10 seconds and poured onto Mitis-Salivarius agar (MS, Gibco, Tokyo, Japan) plates for total Streptococci, improved Mitis-Salivarius agar plates containing 0.02 M bacitracin (Wako, Osaka, Japan) (MSB) and $2 \mathrm{ug} / \mathrm{ml}$ of Gramidine ${ }^{11)}$ for mutans streptococci and Rogosa SL agar plates (Nippon Becton Dickinson Company, Ltd., Tokyo, Japan) for Lactobacilli using an EDDY JET spiral system (Gunze Sangyo, Inc., Tokyo, Japan). In the improved MSB plate, the growth of the mutans streptococci are higher than that in the conventional MSB plate ${ }^{12}$. All samples were then incubated for 48 hours anaerobically. After the anaerobic incubation, we counted the colonies on each agar plate and calculated the number of bacteria per $\mathrm{m} l$ whole-saliva.

\section{Questionnaires}

Questionnaires were distributed by mail with the announcement of school participation and collected at preschool medical check-ups. The questionnaires consisted of five items concerning fluoride usage and diet. Fluoride usage was evaluated by daily usage of fluoride containing dentifrices (yes or no), the experience of fluoride varnish at private dental office or usual health care check-ups (yes, experienced or never) and daily use of mouthwash with fluoride (yes, experienced or never). The questionnaire on 
Table 1 Descriptive analysis of each factors

\begin{tabular}{lccc}
\hline & This study & National average in Japan \\
\hline & Mean & SD & 2.02 \\
Number of decayed teeth & 1.25 & 2.19 & 2.37 \\
Number of filled teeth & 2.90 & 3.26 & 4.38 \\
Number of df teeth & 4.15 & 4.01 & - \\
Salivary flow $(\mathrm{m} l / 3$ mins $)$ & 2.96 & 1.74 & - \\
Salivary pH & 7.276 & 0.198 & - \\
Total Streptococci $\left(\mathrm{CFU} / \mathrm{m} l, \log _{10}\right.$ count) & 6.887 & 0.261 & - \\
Lactobacillus $\left(\mathrm{CFU} / \mathrm{m} l, \log _{10} \operatorname{count}\right)$ & 2.561 & 2.35 & - \\
mutans streptococci $\left(\mathrm{CFU} / \mathrm{m} l, \log _{10}\right.$ count) & 4.208 & 2.305 & - \\
\hline
\end{tabular}

Mean and standard deviation of the data from the oral examination and bacterial cultures obtained in this study. The mean number of decayed or filled teeth was below the average of a national survey in Japan. The data of the national average of Japan were obtained from The Survey of Dental Diseases by Health Policy Bureau Ministry of Health and Welfare Japan (1999).

diet sugar intake consisted of two items, the number of daily intakes of sweet juice and the daily intake of sweet snacks (once, twice, three times or more than four times).

\section{Statistical analysis}

Before the analysis, patients were divided into two groups: subjects free from dental caries and subjects with at least one decayed or filled tooth (df teeth). For the high risk children, subjects were divided into two groups by the $75^{\text {th }}$ percentile of the distribution by of the df teeth. As with microbiological factors, the bacteriological counts were $\log _{10}$-transformed prior to statistical analysis to normalize the variances. After evaluation of the distribution, amount of the mutans streptococci and Lactobacillus were categorized into four groups by the $25^{\text {th }}, 50^{\text {th }}$ and $75^{\text {th }}$ percentile of the distribution.

Logistic regression analysis was used to evaluate the crude or adjusted odds ratios and their associated 95 percent confidence intervals. To eliminate the confounding factors, multiple Logistic regression analysis was used for factors correlated to the prevalence of dental caries. To confirm the doseresponse relationships, the final factors correlated with the prevalence of the dental caries were classified according to the distribution.

Then two-way ANOVA was used to investigate the co-effect for the mutans streptococci and Lactobacillus for the df teeth.

\section{Results}

Forty-two percent of the children participating in
Table 2 Results of the data obtained from questionnaires in this study

\begin{tabular}{lrr}
\hline & $\mathrm{n}$ & $\%$ \\
\hline Usage of fluoride containing dentifrice & & \\
Yes & 404 & $61.1 \%$ \\
No & 245 & $37.1 \%$ \\
Experience of fluoride varnish & & \\
Regularly & 382 & $57.8 \%$ \\
Experienced & 235 & $35.6 \%$ \\
Never & 33 & $5.0 \%$ \\
Regularly & & \\
Experienced & 38 & $5.7 \%$ \\
Never & 108 & $16.3 \%$ \\
Experience & 495 & $74.9 \%$ \\
Once & & \\
Twice & 325 & $49.2 \%$ \\
Three times & 228 & $34.5 \%$ \\
Four or more times & 79 & $12.0 \%$ \\
Sweet soft drink intake (daily) & 17 & $2.6 \%$ \\
Once & & \\
Twice & 34 & $5.1 \%$ \\
Three times & 393 & $59.5 \%$ \\
Four or more times & 198 & $30.3 \%$ \\
& 24 & $3.7 \%$ \\
\hline Sweet snack & & \\
& & \\
& &
\end{tabular}

this study had decayed teeth, and $73.1 \%$ had df teeth. The distribution of results is shown in Fig. 1. Table 1 shows the mean and SD of the number of the decayed, filled, and df (decayed or filled) teeth, and the salivary flow, salivary $\mathrm{pH}$ and salivary levels of the bacteria investigated in this study. Table 2 shows the categorized results from questionnaires. Salivary levels of mutans streptococci were not detected 
(A)

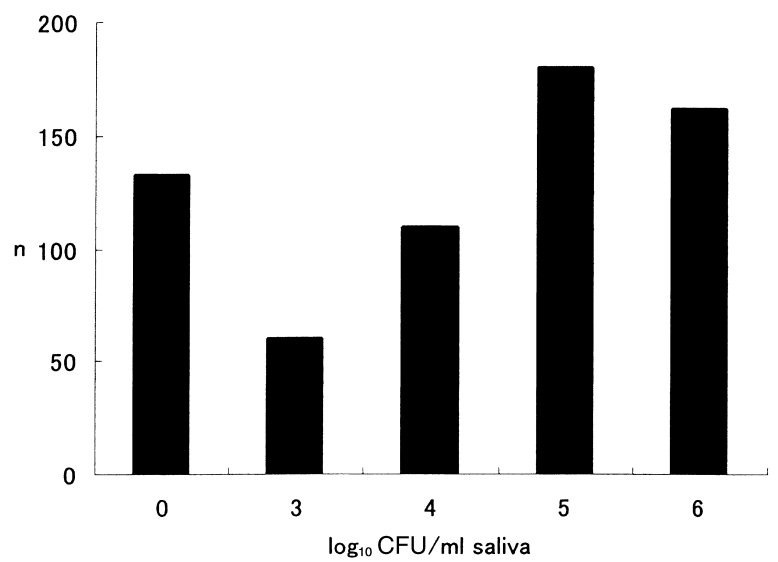

(B)

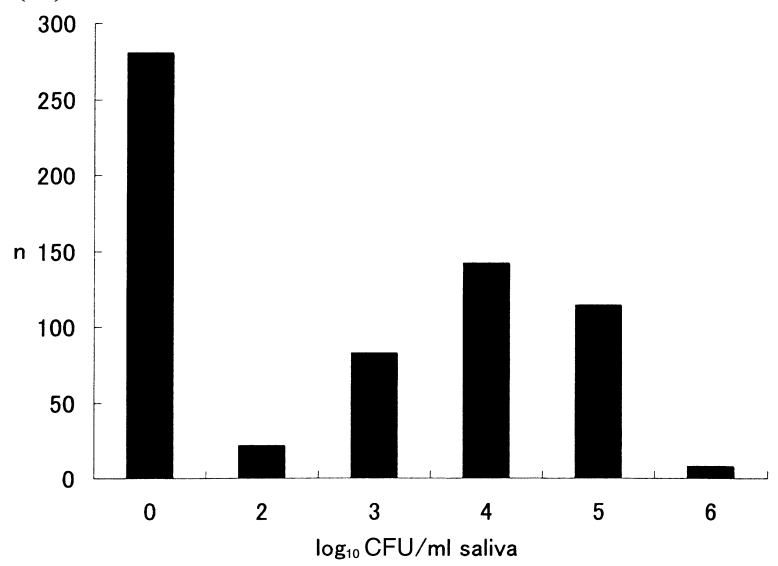

Fig. 2 The distribution of the microflora

The distribution of the mutans streptococci (A) and Lactobacillus (B) in this study. $20.6 \%$ of children could not detected mutans streptococci from their saliva, however, from $25.1 \%$ of children, mutans streptococci could be detected more than $10^{6} \mathrm{CFU} / \mathrm{m} l \mathrm{saliva}$.

(A)

Table 3 The odds ratios for the dental caries

\begin{tabular}{lcccccc}
\hline & $\begin{array}{c}\text { Crude } \\
\text { odds ratio }\end{array}$ & $95 \% \mathrm{CI}$ & $P$-value & $\begin{array}{c}\text { Adjusted } \\
\text { odds ratio }\end{array}$ & 95\% CI & $P$-value \\
\hline Salivary flow rate & 0.753 & $0.605-0.936$ & 0.011 & 0.866 & $0.677-1.107$ & 0.249 \\
Salivary pH & 0.607 & $0.250-1.477$ & 0.272 & & & \\
Lactobacillus ( $\log _{10}$ count) & 1.643 & $1.449-1.864$ & $<0.001$ & 1.405 & $1.222-1.617$ & $<0.001$ \\
Total Streptococci $\left(\log _{10}\right.$ count) & 1.835 & $1.334-2.523$ & $<0.001$ & 1.156 & $0.806-1.658$ & 0.431 \\
mutans streptococci ( $\log _{10}$ count) & 1.406 & $1.297-1.525$ & $<0.001$ & 1.246 & $1.135-1.368$ & $<0.001$ \\
Dentifrice containing fluoride & 0.837 & $0.585-1.196$ & 0.328 & & & \\
Fluoride varnish & 1.343 & $0.570-3.164$ & 0.500 & & & \\
Fluoride mouth rinse & 0.500 & $0.205-1.219$ & 0.127 & & & \\
Juice intake & 1.409 & $1.107-1.794$ & 0.005 & 1.236 & $0.950-1.607$ & 0.114 \\
Sweet snack intake & 1.106 & $0.839-1.459$ & 0.475 & & & \\
\hline
\end{tabular}

(B)

\begin{tabular}{lccccc}
\hline & $\begin{array}{c}\text { Crude } \\
\text { odds ratio }\end{array}$ & $95 \%$ CI & $P$-value & $\begin{array}{c}\text { Adjusted } \\
\text { odds ratio }\end{array}$ & $95 \%$ CI \\
\hline Salivary flow rate & 0.640 & $0.410-0.999$ & 0.049 & 0.651 & $0.402-1.054$ \\
Salivary pH & 0.323 & $0.086-1.206$ & 0.093 & & 0.081 \\
Lactobacillus $\left(\log _{10} \operatorname{count}\right)$ & 1.566 & $1.290-1.900$ & $<0.001$ & 1.342 & $1.082-1.663$ \\
Total Streptococci $\left(\log _{10}\right.$ count) & 1.821 & $1.158-2.864$ & 0.009 & 1.197 & $0.723-1.981$ \\
mutans streptococci $\left(\log _{10}\right.$ count) & 1.771 & $1.354-2.315$ & $<0.001$ & 1.378 & $1.029-1.874$ \\
Dentifrice containing fluoride & 0.879 & $0.506-1.527$ & 0.647 & & 0.032 \\
Fluoride varnish & 1.031 & $0.666-1.595$ & 0.892 & & \\
Fluoride mouth rinse & 0.521 & $0.215-1.256$ & 0.150 & & \\
Juice intake & 1.247 & $0.915-1.700$ & 0.163 & & \\
Sweet snack intake & 1.251 & $0.830-1.884$ & 0.284 & &
\end{tabular}

Crude and multivariate adjusted odds ratios for subjects with df teeth or not (A) and for the high risks (B). Odds ratios were calculated by Logistic regression analysis. $P$-values were calculated by the Wald test. Among the 10 factors investigated in this study, only two factors such as salivary levels of mutans streptococci and Lactobacillus had statistically significant correlation with the dental caries conditions. 
(A)

Table 4 Dose-response relationships of the odds ratios

\begin{tabular}{ccccccccc}
\hline & \multicolumn{4}{c}{ with or without dental caries } & \multicolumn{2}{c}{ high risks } \\
\hline & $\mathrm{n}$ & Odds ratio & $95 \% \mathrm{CI}$ & $P$-value & Odds ratio & $95 \%$ CI & $P$-value \\
\hline 0 & 280 & reference group & & reference group & \\
$0<\log _{10} \mathrm{CFU} / \mathrm{m} l<10^{4}$ & 103 & 3.112 & $1.809-5.355$ & $<0.001$ & 1.938 & $1.003-3.746$ & 0.049 \\
$10^{4}<\log _{10} \mathrm{CFU} / \mathrm{m} l<10^{5}$ & 141 & 4.057 & $2.429-6.774$ & $<0.001$ & 4.331 & $2.515-7.457$ & $<0.001$ \\
$10^{5}<\log _{10} \mathrm{CFU} / \mathrm{m} l$ & 121 & 6.812 & $3.587-12.938$ & $<0.001$ & 6.327 & $3.665-10.992$ & $<0.001$ \\
\hline
\end{tabular}

(B)

\begin{tabular}{ccccccccc}
\hline & \multicolumn{4}{c}{ with or without dental caries } & \multicolumn{3}{c}{ high risks } \\
\hline & $\mathrm{n}$ & Odds ratio & $95 \% \mathrm{CI}$ & $P$-value & Odds ratio & $95 \%$ CI & $P$-value \\
\hline 0 & 133 & reference group & & reference group & \\
$0<\log _{10} \mathrm{CFU} / \mathrm{m} l<10^{5}$ & 170 & 2.824 & $1.753-4.549$ & $<0.001$ & 1.139 & $0.552-2.315$ & 0.725 \\
$10^{5}<\log _{10} \mathrm{CFU} / \mathrm{m} l<10^{6}$ & 180 & 3.651 & $2.248-5.929$ & $<0.001$ & 2.063 & $1.060-4.015$ & 0.033 \\
$10^{6}<\log _{10} \mathrm{CFU} / \mathrm{m} l$ & 162 & 11.746 & $6.162-22.390$ & $<0.001$ & 5.649 & $2.981-10.706$ & $<0.001$ \\
\hline
\end{tabular}

Dose-response relationships of the salivary levels of Lactobacillus (A) and mutans streptococci (B) for dental caries. Clear doseresponse relationships were observed in this study.

Table 5 Cross table of the number of df teeth by the salivary levels of mutans streptococci and Lactobacillus

\begin{tabular}{|c|c|c|c|c|c|c|c|}
\hline & & & & & mutans streptococci & & \\
\hline & & & 0 & $0<\log _{10} \mathrm{CFU} / \mathrm{m} l<10^{5}$ & $10^{5}<\log _{10} \mathrm{CFU} / \mathrm{m} l<10^{6}$ & $10^{6}<\log _{10} \mathrm{CFU} / \mathrm{ml}$ & Total \\
\hline \multirow{10}{*}{ 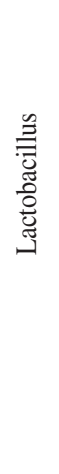 } & \multirow{2}{*}{0} & mean $\pm \mathrm{SD}$ & $1.943 \pm 2.917$ & $2.471 \pm 2.917$ & $3.303 \pm 3.371$ & $4.048 \pm 3.498$ & $2.586 \pm 3.148$ \\
\hline & & $\mathrm{n}$ & 107 & 85 & 67 & 21 & 280 \\
\hline & \multirow{2}{*}{$0<\log _{10} \mathrm{CFU} / \mathrm{m} l<10^{4}$} & mean $\pm \mathrm{SD}$ & $3.250 \pm 2.990$ & $3.447 \pm 3.244$ & $4.108 \pm 3.478$ & $7.077 \pm 5.327$ & $4.147 \pm 3.820$ \\
\hline & & $\mathrm{n}$ & 8 & 45 & 37 & 13 & 103 \\
\hline & \multirow{2}{*}{$10^{4}<\log _{10} \mathrm{CFU} / \mathrm{m} l<10^{5}$} & mean $\pm \mathrm{SD}$ & $3.636 \pm 4.501$ & $4.516 \pm 4.007$ & $4.578 \pm 3.621$ & $7.250 \pm 3.832$ & $5.434 \pm 4.058$ \\
\hline & & $\mathrm{n}$ & 11 & 32 & 47 & 51 & 141 \\
\hline & \multirow{2}{*}{$10^{5}<\log _{10} \mathrm{CFU} / \mathrm{m} l$} & mean $\pm \mathrm{SD}$ & $2.857 \pm 2.642$ & $6.000 \pm 3.640$ & $5.103 \pm 3.880$ & $7.080 \pm 4.341$ & $6.277 \pm 4.276$ \\
\hline & & $\mathrm{n}$ & 7 & 8 & 29 & 75 & 121 \\
\hline & \multirow{2}{*}{ Total } & mean $\pm \mathrm{SD}$ & $2.212 \pm 3.070$ & $3.280 \pm 3.410$ & $4.096 \pm 3.610$ & $6.726 \pm 4.312$ & $4.139 \pm 3.989$ \\
\hline & & $\mathrm{n}$ & 133 & 170 & 180 & 162 & 645 \\
\hline
\end{tabular}

Dose-response relationship was observed by the mutans streptococci and Lactobacillus for df teeth. By two-way ANOVA analysis, $P$-values for the mutans streptococci was $<0.001$ and Lactobacillus $<0.001$. However, $P$-values for the mutans streptococci* Lactobacillus was 0.769 , so interaction of the mutans streptococci and Lactobacillus for the number of df teeth was not observed in this study.

from $20.6 \%$ of the children, and salivary levels of Lactobacillus were not detected from $43.4 \%$ of the children (Fig. 2). However, more than $10^{6} \mathrm{CFU} / \mathrm{ml}$ salivary mutans streptococci were detected from $25.1 \%$ of children.

To correlate the prevalence of the number of $\mathrm{df}$ teeth and risk factors investigated in this study, we performed Logistic regression analysis to calculate the odds ratio for $\mathrm{df}$ teeth. Table 3 shows the result of the crude odds ratios. Among 10 factors investigated in this study, salivary flow, amount of Lactobacillus, amount of total Streptococci, amount of mutans streptococci and daily number of sweet soft drinks taken were correlated with the prevalence 
of dental caries. To eliminate the confounding factors, we performed multivariate Logistic regression analysis using the six factors that correlated with the dental caries. Table 3 shows the results of multivariate Logistic regression analysis. Only two of the five factors, amounts of mutans streptococci and Lactobacillus, were correlated with the prevalence of dental caries. For the medians of the decayed teeth, multivariate adjusted odds ratio were 1.259 by Lactobacillus and 1.105 by mutans streptococci; for the medians of the decayed or filled teeth, were 1.236 by Lactobacillus and 1.160 by mutans streptococci. The same tendencies were observed in the crude odds ratio if the children were divided into groups with or without decayed teeth or divided into two groups by the $75^{\text {th }}$ percentile of the decayed or filled teeth (Table 3-B). To confirm the dose-response relationships, we categorized these factors by the distribution and then performed Logistic analysis again. Table 4 shows the dose-response relationships for these factors. Clear dose-response relationships were observed for the salivary levels of mutans streptococci and Lactobacillus. Then to check the coeffect of the mutans streptococci and Lactobacillus for dental caries, two-way ANOVA analysis was carried out. As shown in Table 5, no co-effects were found in mutans streptococci and Lactobacillus for dental caries.

\section{Discussion}

The etiology of dental caries has been suggested to be classified into three main categories, and this has been confirmed by laboratory investigation and clinical studies. In particular, mutans streptococci and Lactobacillus have been intensively studied as microflora factors. The nature of the acid production and biofilm formation by cariogenic microorganism has been studied ${ }^{13,14)}$. However, clinical studies have shown that some subjects have decayed teeth even though these bacteria were below detection levels in the oral cavity ${ }^{15,16)}$. These results have shown that the etiology of the dental caries cannot be explained easily. In contrast, some studies have shown that, as a substrate factor, dietary intervention to restrict the sucrose consumption has reduced the prevalence or incidence of the dental caries ${ }^{6}$. However, in this study, no correlation was found in daily sucrose consumption and the prevalence of the decayed teeth, filled teeth or df teeth. This may be because the method of surveying sucrose consumption by questionnaire does not reflect the actual conditions for the substitute factors. However, in present, there is no other method to survey for these factors.

The difference in the results may thus be due to the method of surveillance.

This study found no correlation in fluoride usage by dentifrice and mouth rinse and varnish. Clearly, fluoride has been used to reduce the prevalence of dental caries. In this study, the institutions that applied the fluoride were varied. This may also be due to the methods of the investigation. The method of the fluoride application and intervals of application may be reflected in the result.

Some studies have shown that salivary flow rate and salivary buffering capacity or salivary $\mathrm{pH}$ contribute to the incidence of dental caries ${ }^{17,18)}$, while others found no correlation between these factors and dental caries ${ }^{16,19}$. In this study, we could not find any statistically significant correlation between dental caries prevalence and salivary factors. It is generally considered that salivary flow rate was affected by the side effects of medication or systemic diseases. In general, the salivary flow rate of children is high, and few children take drugs affecting the sympathetic nerve system and reducing the salivary flow rate. Sgan-Cohen et al. found a significant correlation of the salivary flow and dental caries, however the correlation was weaker for other factors such as microflora ${ }^{21)}$. This may be the main reason for the contradictory result.

In conclusion, of the three main factors suggested by Keyes, the microflora factors are strongly correlated with the prevalence of dental caries for the preschool children in Japan. In the future, controlling this factor for the cohort may lead to strong strategies for preventing dental caries through community-based prevention programs.

\section{References}

1) Keyes, P.H.: Present and future measures for dental caries control. J Am Dent Assoc 79: 1395-1404, 1969.

2) Seow, W.K.: Biological mechanisms of early childhood caries. Community Dent Oral Epidemiol 26: 8 27, 1998

3) Clarkson, J.J. and McLoughlin, J.: Role of fluoride in oral health promotion. Int Dent J 50: 119-128, 2000.

4) Sundin, B. and Granath, L.: Sweets and other sugary products tend to be the primary etiologic factors in dental caries. Scand J Dent Res 100: 137-139, 1992.

5) Newbrun, E.: Sugar and dental caries. Clin Prev 
Dent 4: 11-14, 1982.

6) Karjalainen, S., Sewon, L., Soderling, E., Lapinleimu, H., Seppanen, R. and Simell, O.: Oral health of 3year-old children and their parents after 29 months of child-focused antiatherosclerotic dietary intervention in a prospective randomized trial. Caries Res 31: 180-185, 1997.

7) Makinen, K.K., Bennett, C.A., Hujoel, P.P., Isokangas, P.J., Isotupa, K.P. and Pape, H.R.: Xylitol chewing gums and caries rates: a 40-month cohort study. $J$ Dent Res 74: 1904-1913, 1995.

8) Takeuchi, H., Senpuku, H., Matin, K., Kaneko, N., Yusa, N., Yoshikawa, E., Ida, H., Imai, S., Nishizawa, T., Abei, Y., Kono, Y., Ikemi, T., Toyoshima, Y., Fukushima, K. and Hanada, N.: New dental drug delivery system for removing mutans streptococci from the oral cavity: effect on oral microbial flora. Jpn J Infect Dis 53: 211-212, 2000.

9) World Health Organization: Oral Health Survey: Basic Methods. 4th ed. World Health Organization, Geneva, 1997.

10) Hanada, N., Nomura, Y., Takeuchi, H., Senpuku, H., Ida, H., Yoshikawa, E. and Kumagai, T.: New dental drug delivery system for removing mutans streptococci. J Dent Res 80: 567, 2001.

11) Suzuki, T., Tagami, J. and Hanada, N.: Role of F1F0ATPase in the growth of Streptococcus mutans GS5. J Appl. Micro Boil 8: 555-562, 2000.

12) Hanada, N., Imai, S., Nishizawa, T. and Mukasa, H.: Clinical Biology of the mutans streptococci. Quintessence Co., Tokyo, 2003, pp.90-97. (in Japanese)

13) de Soet, J.J., Nyvad, B. and Kilian, M.: Strainrelated acid production by oral streptococci. Caries Res 34: 486-490, 2000.

14) Rozen, R., Bachrach, G., Bronshteyn, M., Gedalia,
I. and Steinberg, D.: The role of fructans on dental biofilm formation by Streptococcus sobrinus, Streptococcus mutans, Streptococcus gordonii and Actinomyces viscosus. FEMS Microbiol Lett 195: 205-210, 2001.

15) Alaluusua, S., Kleemola-Kujala, E., Gronroos, L. and Evalahti, M.: Salivary caries related test as predictors of future caries increment in teenagers. Oral microbiology and immunology 5: 77-81, 1990.

16) Gabris, K., Nagy, G., Madlena, M., Denes, Z., Marton, S., Keszthelyi, G. and Banoczy, J.: Associations between microbiological and salivary caries activity tests and caries experience in Hungarian adolescents. Caries Res 33: 191-195, 1999.

17) Holbrook, W.P.: Dental caries and cariogenic factors in pre-school urban Icelandic children. Caries Res 27: 431-437, 1993.

18) Sundin, B., Granath, L. and Birkhed, D.: Variation of posterior approximal caries incidence with consumption of sweets with regard to other cariesrelated factors in 15-18-year-olds. Community Dent Oral Epidemiol 20: 76-80, 1992.

19) Dodds, M.W., Johnson, D.A., Mobley, C.C. and Hattaway, K.M.: Parotid saliva protein profiles in caries-free and caries-active adults. Oral Surg Oral Med Oral Pathol Oral Radiol Endod 83: 244-251, 1997.

20) Pattanaporn, K. and Navia, J.M.: The relationship of dental calculus to caries, gingivitis, and selected salivary factors in 11- to 13-year-old children in Chiang Mai, Thailand. J Periodontol 69: 955-961, 1998.

21) Sgan-Cohen, H.D., Steinberg, D., Zusman, S.P. and Sela, M.N.: Dental caries and its determinants among recent immigrants from rural Ethiopia. Community Dent Oral Epidemiol 20: 338-342, 1992. 